\title{
Pulsations in hot supergiants
}

\author{
Melanie Godart ${ }^{1}$, Arlette Grotsch-Noels ${ }^{2}$ and Marc-Antoine Dupret ${ }^{2}$ \\ ${ }^{1}$ Dept. of Astronomy, University of Tokyo, Japan \\ email: melanie.godart@gmail.com \\ ${ }^{2}$ Dept. of Astrophysics, Geophysics and Oceanography, University of Liège, Belgium
}

\begin{abstract}
Massive stars are the cosmic engines that shape and drive our Universe. Many issues such as their formation, their stability and the mass loss effects, are far from being completely understood. Recent ground-based and space observations have shown pulsations in massive MS and post-MS stars, such as acoustic and gravity modes excited by the $\kappa$-mechanism and even solar-like oscillations. Theoretical studies emphasized the presence of strange modes in massive models, and recent theoretical analyses have shown that hot supergiants can pulsate in oscillatory convective modes. We review the instability domains of massive stars as well as their excitation mechanisms and present the latest results.
\end{abstract}

Keywords. stars: early-type, stars: oscillations, supergiants, Wolf-Rayet

\section{Introduction}

Hot supergiants comprise massive stars in different evolutionary states, including $\mathrm{O}$ and B supergiants, Luminous Blue Variables (LBV) and Wolf-Rayet (WR) stars. Periodic variability has been detected in such stars with a possible link to pulsations. This review summarizes the theoretical efforts in the field of massive star pulsations. Massive stars pulsate in regular and strange modes. Regular modes are excited with periods around hours for the $\beta$ Cephei type modes (low-order $\mathrm{p}$ and g modes) and with longer periods $(\sim$ days) for Slowly Pulsating B (SPB) type modes (high-order g modes). Strange modes are excited in a larger range of periods, of the order of hours or days. Several driving mechanisms can be responsible for the pulsations: the $\epsilon$-mechanism, the $\kappa$-mechanism and strange-mode instabilities. Moreover, Belkacem et al. (2009) reported the presence of solar-like oscillations in $\beta$ Cephei stars. Both the convective region associated with the metal $(Z)$ opacity bump and the convective core are found to efficiently drive acoustic modes in $10 M_{\odot}$ models (Belkacem et al. 2010). Their results have been reinforced by observations since Degroote et al. (2010) have detected solar-like oscillations in a massive main-sequence (MS) star observed by CoRoT.

\section{Regular modes in massive stars}

Though the instabilities due to the $\epsilon$-mechanism have been extensively studied in the framework of massive stars (Simon \& Stothers 1969, Ziebarth 1970), this mechanism was later neglected in favor of stronger instabilities produced by the $\kappa$-mechanism and strange-mode instabilities. However, recently 19 significant frequencies have been observed in the B supergiant Rigel (Moravveji et al. 2012a), with periods ranging from 1 to 75 days. Moravveji et al. (2012b) suggest that these modes are excited by the $\epsilon$-mechanism occurring in the hydrogen-burning shell.

Less massive stars such as SPB and $\beta$ Cephei stars are pulsating due to the $\kappa$ mechanism activated by the $Z$ opacity bump at $\log T \sim 5.2$. Their instability domains are well known to this date thanks to the works of Pamyatnykh (1999) and later with the 

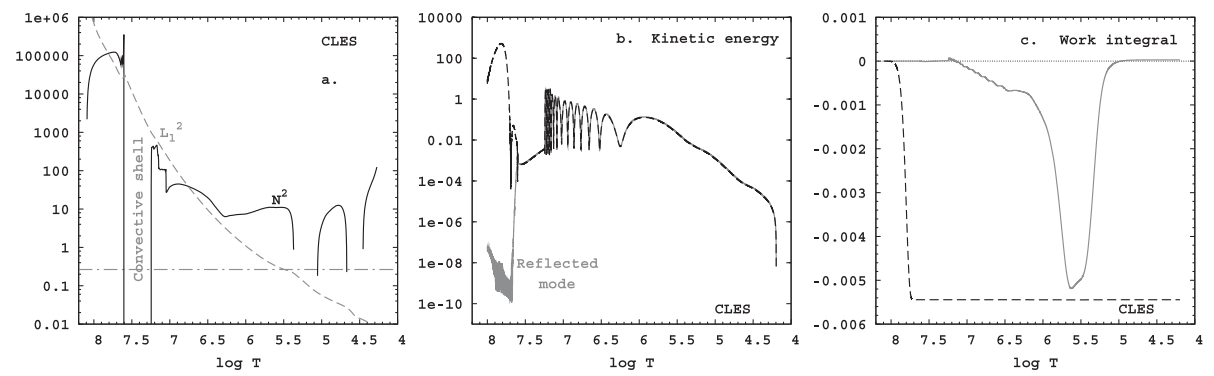

Figure 1. a) Dimensionless Brunt-Väisälä (solid line) and Lamb frequencies (dashed line) as a function of $\log T$ in an $18 M_{\odot}$ post-MS model. $b$ ) Kinetic energy of 2 modes of $\omega=0.52 . c$ ) Work integral for the same modes. The reflected mode is excited (solid line) while the mode entering the radiative core is damped (dashed line) (Godart 2011).

new OP opacities (Seaton et al. 1994) and the new abundances (Asplund et al. 2005), thanks to Miglio (2007), Miglio et al. (2007), and Pamyatnykh (2007). The computations were limited to the MS and/or to the lower masses (up to $20 M_{\odot}$ ). However, the instability domain of these stars could be extended to larger masses (e.g. Kiriakidis et al. 1992, Moskalik \& Dziembowski 1992). One reason for the limitation of the computation is perhaps the following: Post-MS massive stars consist of a dense pure helium core surrounded by a large hydrogen envelope. The high density contrast between both regions causes a large Brunt-Väisälä frequency, producing (1) a large number of oscillations around the large Brunt-Väisälä frequency, generating possible numerical issues; and (2) a strong radiative damping in the very dense core, which should cancel the occurrence of $\mathrm{g}$ modes (see e.g. Eq. 14 of Godart et al. 2009). However, in the mid-2000s, new observations contradicted these assumptions. Indeed, periodic variabilities were detected in B supergiant stars (Saio et al. 2006, Lefever et al. 2007) attributed to non-radial g mode pulsations. In addition, Saio et al. (2006) provided an explanation for the presence of $\mathrm{g}$ modes in such post-MS massive stars: an intermediate convective zone (ICZ) surrounds the pure helium core and prevents the g modes from entering the core. The authors showed that in that case, the $\kappa$-mechanism activated by the $Z$ opacity bump is sufficient to excite the g modes. This effect was later investigated by Gautschy et al. (2009) and Godart et al. (2009). These authors showed that the presence of an ICZ depends on the past history of the star, since it is produced thanks to the occurrence of a region of neutrality of the temperature gradients (semi-convection) during the MS. Moreover, some processes, such as mass loss and overshooting or the Ledoux criterion for convection, are able to prevent the formation of the semi-convective region on the MS and, therefore, to suppress the ICZ and the occurrence of g modes on the post-MS (Godart et al. 2009, Lebreton et al. 2009). We show in Figs. 1a-c the study (propagation diagram, kinetic energy and work integral) of two modes of close frequencies for an $18 M_{\odot}$ post-MS model. The stable mode crosses the convective barrier and presents a high amplitude in the stellar core, illustrated by a large kinetic energy (Fig. 1b, black dashed line). Hence, this mode suffers a strong damping in the core $(\log T \sim 7.8)$, much more efficient than the $\kappa$-mechanism in the superficial layers (see Fig. 1c, representing the work integral of the mode). On the other hand, the unstable mode (grey solid line) is reflected on the convective shield: the amplitude is very small in the stellar core. This mode is excited by the $\kappa$-mechanism at $\log T \sim 5.3$ (see the work integral).

Figure 2 shows the instability domains for massive stars of Saio (2011) (left panel) and Godart et al. (2011) (right panel). Low-order p and strange modes (black dashed lines) 

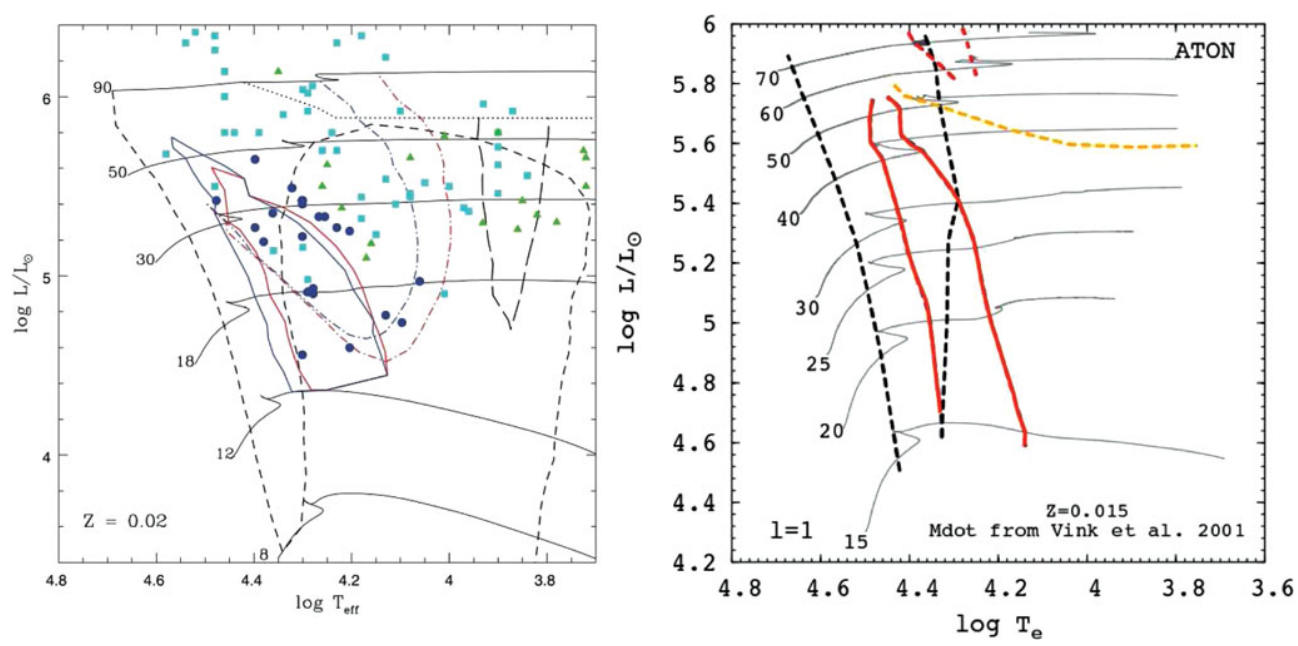

Figure 2. Left: Instability domains for various types of modes (Saio 2011, fig. 1, by permission of Oxford University Press on behalf of The Royal Astronomical Society). The low-order p and strange modes are shown in black dashed lines, the boundaries becoming horizontal due to strange-mode instability. Solid red and blue lines stand for the g modes $(l=1$ and $l=2$, respectively). Right: $\kappa$-mechanism instability domains in the HR diagram (Godart 2011). The $l=1$ instability domains for low-order $\mathrm{p}$ and g modes $\left(15\right.$ to $\left.30 M_{\odot}\right)$ and for low-order $\mathrm{p}$ and g modes and adiabatic strange modes $\left(40\right.$ to $\left.70 M_{\odot}\right)$ are shown in the black dashed lines. Solid red lines stand for the high-order g modes.

are found to be excited on the MS and the post-MS (the strange modes are excited in the higher luminosity models only), while high-order g modes are excited in the postMS phase. Recently, Ostrowski et al. (2012) and Daszyńska-Daszkiewicz et al. (2013) reported that the ICZ is not a necessary condition for the g mode detection. They point out that a minimum in the Brunt-Väisälä frequency is sufficient for forcing the trapping of the $g$ mode outside the radiative damping core. The computations were performed with the Ledoux criterion for convection and the mean molecular weight gradient prevents therefore the ICZ to form in the $16 M_{\odot}$ model. The minimum in the Brunt-Väisälä frequency is associated with the change of the temperature gradient from the adiabatic to the radiative one above the semi-convective region. Note that for larger masses, the ICZ should appear, though it will stay smaller than when taking the Schwarzschild's convection treatment.

\section{Strange modes}

In addition to the usual spectrum of regular modes, other instabilities are also responsible for the pulsations in massive stars. The term strange modes was put forward by Cox et al. (1980) because of the strange behaviour of these modes in a modal diagram: it consists in the plot of the mode frequency (normalized by the dynamical time $\sqrt{R^{3} / G M}$ ) as a function of a stellar parameter, e.g. $T_{\text {eff }}$ or $M$, for a homogeneous set of models, e.g. ZAMS models or pure He stars. The variation of the regular p-mode frequency scales as $\sqrt{G M / R^{3}}$. Nonetheless, due to their confinement in a small cavity, strange modes present a very different behaviour. Indeed, the dimensionless frequency, $\omega$, depends on the size of the mode propagation cavity: $\omega \sim n \pi \sqrt{\left(R^{3} / G M\right)}\left(\int_{\text {cavity }} c_{s}^{-1} d r\right)$, which means that the dimensionless frequency of a strange mode decreases compared to that of a regular mode if the size of the cavity increases more rapidly than the radius of 


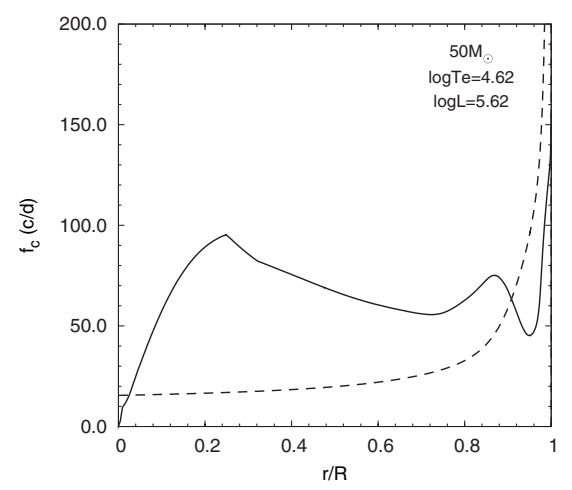

Figure 3. Critical frequency of radial modes (solid line) in $50 M_{\odot}$ MS model. The dashed line stands for $f_{t}$, the inverse of the time needed for an acoustic wave to travel from $r$ to the surface and back (Godart 2011).

the star. This is the case on the MS, i.e. with decreasing $T_{\text {eff }}$ or increasing age, or for a homogeneous set of models of different masses. Figure 2 of Glatzel \& Kiriakidis (1993a) shows a modal diagram in which adiabatic and non-adiabatic strange modes (see below for the definitions) are visible, with decreasing frequencies. Reviews of strange modes may be found in, e.g., Saio et al. (1998), Noels et al. (2008), Glatzel (2009), Saio (2009) and Godart (2011). Strange modes are found to be present in various types of stars and mass ranges, such as the pure He stars, low mass supergiants, massive main-sequence stars, evolved massive stars, central stars of planetary nebulae, classical Cepheids, and Wolf-Rayet stars. The phenomenon of strange modes is not even restricted to stellar objects: accretion disks can also be unstable due to strange modes (Glatzel \& Mehren 1996).

Adiabatic and nonadiabatic strange modes: By limiting our discussion to the radial adiabatic strange modes (see below), we can easily understand their origin. Figure 3 shows the angular critical frequency for radial modes, roughly given by $c_{s} / 2 H_{p}$, as a function of the stellar radius. In low- and intermediate-mass stars, the cavity created by this critical frequency extends over the whole star. However, with increasing mass, a local minimum appears in the superficial layers, and therefore a new propagating cavity. This cavity (which appears for masses above $\sim 40 M_{\odot}$ ) comprises a new set of modes: the strange modes. A large contribution of radiation pressure to the total pressure in addition to the proximity of opacity bumps are necessary to produce the cavity. Indeed, these conditions create a density and hence a sound-speed inversion. The information which could be obtained from strange modes is thus related to that superficial cavity, in particular to the sound speed and thus the temperature and the density profiles. For more details and for the determination of this critical frequency see, e.g., Saio et al. (1998) and Godart (2011). Though the critical frequency for non-adiabatic strange modes is different, these modes also propagate in a narrow superficial cavity. The confinement of the modes leads to two major consequences: (1) the dimensionless angular frequencies decrease with increasing mass of the star, explaining the peculiar behaviour of strange modes in a modal diagram, and (2) the modes have a small kinetic energy and therefore a large imaginary part of the eigenfrequency. The imaginary part of an adiabatic strange mode (labelled S1u) is visible in fig. 2 of Glatzel \& Kiriakidis (1993a): it is a few orders of magnitude larger than the imaginary part of regular modes.

Saio et al. (1998) show in their fig. 2 (left panel) two modal diagrams for a set of ZAMS homogeneous models: the adiabatic dimensionless frequency distribution is given 

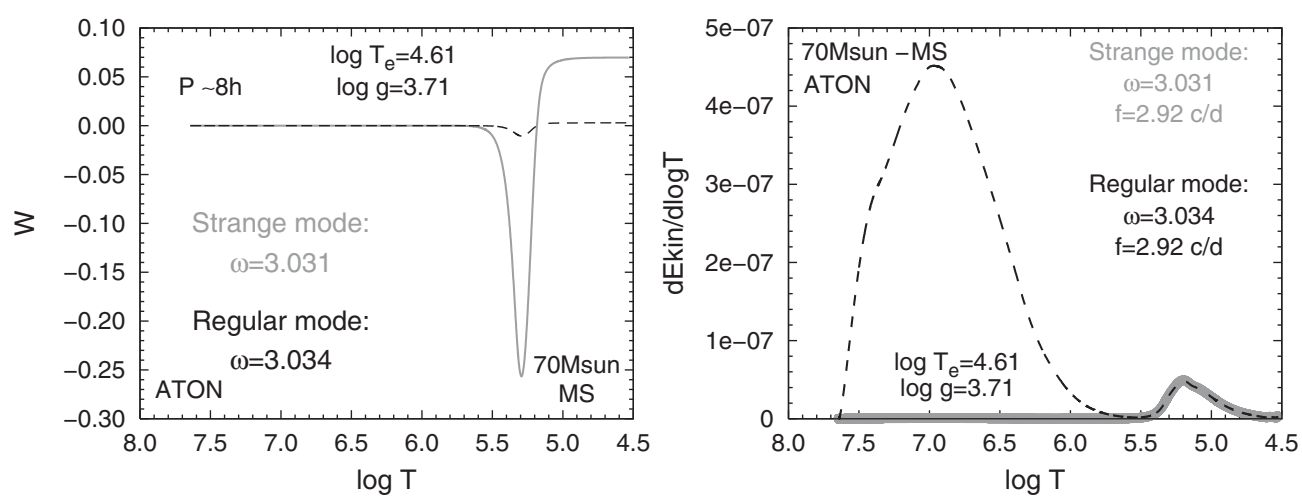

Figure 4. Left: Work integral for two unstable modes: regular mode (dashed line) and strange mode (solid line). The imaginary part of the strange mode is much larger because of the mode trapping. Right: Kinetic energy (surface under the curve) of the same modes. The amplitude confinement of the strange mode is clearly visible (thick grey line) (from Godart 2011).

as a function of the mass in the top panel while the real part of the non-adiabatic frequency is given in the middle panel. A quick look at the top panel allows one to notice the presence of a strange mode (decreasing frequency). Since this panel displays the adiabatic frequencies, the strange modes have an adiabatic counterpart. Regular modes and adiabatic strange modes interact with an avoided crossing.

On the other hand, the middle panel of the same figure presents one or several additional spectra superimposed on the normal mode spectrum: the non-adiabatic strange modes. These modes do not just cross the regular ones but an interaction may occur with an accompanying unfolding of the crossings. The modes coalesce, forcing one of the participating modes to become overstable and the other one to become stable. The real parts of the frequencies of the two interacting modes are essentially identical while their imaginary parts are approximately of the same magnitude but with opposite signs.

Excitation mechanism: Two mechanisms are responsible for the strange mode pulsations: the enhanced $\kappa$-mechanism and the strange-mode instabilities (SMI). The $\kappa$ mechanism (activated by the $Z$ or the He opacity bump) is enhanced by the confinement of the mode in a small region. This driving mechanism accounts for the strange modes with an adiabatic counterpart. The work integral (positive at the surface for unstable modes) and the kinetic energy of a regular and a strange mode are shown in Fig. 4: both modes are excited by the $\kappa$-mechanism due to the $Z$ opacity bump. Figure 4 (right panel) emphasizes the confinement of the strange mode in a superficial region which induces a large inertia and hence a larger growth rate for the strange mode $\left(\eta=W \sigma /\left[4 \pi E_{k}\right]\right)$.

The SMI derives directly from the definition of the strange mode without adiabatic counterpart: one component of the pair is always unstable. Hence, if the conditions for the existence of strange modes are fulfilled (high $L / M$, high radiation pressure), they can always be excited. Glatzel et al. (1993) represent in their fig. 2 the real (top panel) and imaginary (bottom panel) part of the frequencies for a set of pure helium stars. It is evident that the coalescence of two modes coincides with the appearance of two nearly complex conjugate eigenfrequencies.

Prospects: With the addition of a model atmosphere, for example the FASTWIND model atmosphere (Santolaya-Rey et al. 1997, Puls et al. 2005), a temperature inversion may appear in some models generating an atmospheric cavity in which atmospheric strange modes are found excited with periods of the order of days (Godart et al. 2010, 2011). In addition, oscillatory convection modes (Saio 2011) in the g mode period range 
have been found to be present in massive star models with very large growth rates. Saio (2011) computed instability domains for these modes (Fig. 2, left panel, dot-dashed lines) by assuming that they could be observable if the ratio of the photospheric amplitude to the maximum amplitude in the interior is larger than 0.2. Moreover, our preliminary results for the effect of convection on the driving show that the use of time-dependent convection rather than frozen convection still allows these modes to be excited, though the instability is weaker. Sonoi \& Shibahashi (these proceedings) studied the instability of oscillatory convective modes in $60 M_{\odot}$ models, and suggest a link between these instabilities and the lack of stars above the Humphreys-Davidson (HD) limit (Humphreys \& Davidson 1979).

\section{Hot pulsators}

$O$ and $B$ supergiants: Models of $\mathrm{O}$ and $\mathrm{B}$ supergiants show pulsation of low-order $\mathrm{p}$ and $\mathrm{g}$ modes ( $\beta$ Cephei type modes) on the MS, high-order $\mathrm{g}$ modes on the post-MS (SPB type modes) and strange modes on the MS and the post-MS. Variations have been detected in OB and A supergiant stars (Burki et al. 1978, Lovy et al. 1984, Kaufer et al. 1997, Waelkens et al. 1998), and have been suggested to be related to pulsations (Saio et al. 2006, Lefever et al. 2007). Saio et al. (2006) proposed to name this new type of pulsating stars the SPB supergiant stars (SPBsg). Instabilities due to the $\epsilon$-mechanism have been detected (Moravveji et al. 2012a, 2012b) and stochastic oscillations have been observed also (Degroote et al. 2010). Periodic variabilities have been detected in O-type MS stars (e.g. Blomme et al. 2011, Mahy et al. 2011). Furthermore, Aerts et al. (2010) have detected a strange-mode candidate in the B supergiant star HD 50064 from CoRoT photometry. The candidate mode has a period of $37 \mathrm{~d}$, with a sudden amplitude increase of a factor 1.6 occurring once on a timescale of $137 \mathrm{~d}$. Models of this kind of star show strange-mode pulsations with periods of the order of days, though such periods could result from g mode pulsations (Godart 2011).

Luminous Blue Variables ( $L B V$ ): The limits of SMIs were investigated in post-MS models (Glatzel \& Kiriakidis 1993b, Kiriakidis et al. 1993). The instability limits of modes associated with He ionization appear to be similar to the HD limit, while the modes associated with the $Z$ bump give strongly metallicity-dependent instability domains (Kiriakidis et al. 1993). Hence strange modes could be responsible for the violent mass-loss outbursts in highly luminous stars (LBV phenomenon), which was previously explained by a dynamical instability (Stothers \& Chin 1993). However, this dynamical instability could not reproduce the HD limit: it appears at much lower effective temperature and for high metallicity. Glatzel \& Kiriakidis (1993b) then suggested that strange modes could trigger the violent mass-loss event. Their results show good agreement with the HD limit, though this limit is not proven to be related to strange modes yet.

Wolf-Rayet Stars (WR): After an LBV event, massive stars become WR (e.g. Chiosi $\&$ Maeder 1986), also subject to pulsations. Short periods (of order or less than an hour) were attributed to the radial fundamental mode driven by the $\epsilon$-mechanism (Noels \& Gabriel 1981). Since strange modes were theoretically found in pure He stars, these were suggested to be present in WR stars (Glatzel et al. 1993). Longer periods (order of hours) have later been suggested (e.g. Rauw et al. 1996 and references therein), with a possible link to non-radial pulsations (Scuflaire \& Noels 1986, Noels \& Scuflaire 1986). Recently, Lefèvre et al. (2005) observed a longer pulsation period of about 9.8 hours in WR 123 with MOST. Based on the results of Glatzel et al. (1993) who found strange modes with periods shorter than 30 minutes in He-star models for WR, Lefèvre et al. (2005) concluded that the period they discovered was too long to be excited by 
SMI. However, Dorfi et al. (2006) showed that this instability is in fact consistent with a strange-mode pulsation by adding a H-rich envelope to the model in order to increase the radius and therefore the pulsation period. This is however in contradiction with the results of Crowther et al. (1995), who found a very small $\mathrm{H}$ abundance in the envelope of WR 123. Townsend \& MacDonald (2006) suggested that the 9.8 hour period may be attributed to g mode instability occurring in the deep opacity bump, at $\log T \sim 6.3$. In that case, the excited periods range from 10 to 21 hours for late WN (WNL) models and from 3 to 13 hours for early WN (WNE) models, in agreement with Lefèvre et al.'s observed period.

\section{Conclusions}

Massive stars are nowadays surrounded by many mysteries whose implications extend far beyond MS and post-MS phases. With asteroseismology, we have a tool to attempt to unravel these mysteries and improve our understanding of stellar evolution. Observational proof of pulsations in the most massive stars is increasing, and new strategies are emerging, for instance, the use of the macroturbulent broadening affecting the line profiles of massive stars. This extra broadening has been suggested to be produced by pulsation modes (e.g. Lucy 1976, Aerts et al. 2009), and recent observational work seems to support this hypothesis (Simón-Díaz et al. 2010, Simón-Díaz 2011). But the wealth and diversity of new observations require a solid theoretical framework to understand and interpret the physical processes at play. High-order g modes probe the deepest layers and bring information about the mixed regions along with the processes affecting the mixing such as mass loss and overshooting, or even the convective criterion. Strange modes probe the superficial layers and could bring information on the density inversion region and the opacity profile while oscillatory convection modes are related to the convective region properties and the convection treatment to adopt.

\section{Acknowledgements}

MG thanks the science organizing committee of the conference for the invitation. This research has been funded by the Japanese Society for Promotion of Science (JSPS).

\section{References}

Aerts, C., Puls, J., Godart, M., \& Dupret, M.-A. 2009, A\& A, 508, 409

Aerts, C., Lefever, K., Baglin, A., et al. 2010, A\&A, 513, L11

Asplund, M., Grevesse, N., \& Sauval, A. J. 2005, ASP-CS, 336, 25

Belkacem, K., Samadi, R., Goupil, M., et al. 2009, Science, 324, 1540

Belkacem, K., Dupret, M. A., \& Noels, A. 2010, A\&A, 510, A6

Blomme, R., Mahy, L., Catala, C., et al. 2011, A\& $A$, 533, A4

Burki, G., Maeder, A., \& Rufener, F. 1978, A\& $A$, 65, 363

Chiosi, C. \& Maeder, A. 1986, ARA\&A, 24, 329

Crowther, P. A., Smith, L. J., \& Hillier, D. J. 1995, A\& A, 302, 457

Daszyńska-Daszkiewicz, J., Ostrowski, J., \& Pamyatnykh, A. A. 2013, MNRAS, 432, 3153

Degroote, P., Briquet, M., Auvergne, M., et al., 2010, A\& $A$, 519, A38

Dorfi, E. A., Gautschy, A., \& Saio, H. 2006, A\&A, 453, L35

Gautschy, A. 1992, MNRAS, 259, 82

Gautschy, A. 2009, A\&A, 498, 273

Glatzel, W. 2009, CoAst, 158, 252

Glatzel, W. \& Kiriakidis, M. 1993a, MNRAS, 262, 85

Glatzel, W. \& Kiriakidis, M. 1993b, MNRAS, 263, 375 
Glatzel, W. \& Mehren, S. 1996, MNRAS, 282, 1470

Glatzel, W., Kiriakidis, M., \& Fricke, K. J. 1993, MNRAS, 262, L7

Godart, M. 2011, Ph.D. thesis, University of Liège

Godart, M., Noels, A., Dupret, M., \& Lebreton, Y. 2009, MNRAS, 396, 1833

Godart, M., Dupret, M.-A., Noels, A., Aerts, C., \& Simón-Díaz, S. 2010, AN 331, P52

Godart, M., Dupret, M.-A., Noels, A., et al. 2011, in: C. Neiner, G. Wade, G. Meynet \& G. Peters (eds.), Active OB stars: Structure, Evolution, Mass-Loss, and Critical Limits, IAU Symposium No. 272, (Cambridge: Cambridge University Press), p. 503

Grevesse, N. \& Noels, A. 1993, in: B. Hauck, S. Paltani \& D. Raboud, (eds.), Perf. de l'Assoc. Vaud. des Cherch. en Phys., p. 205

Humphreys, R. M. \& Davidson, K. 1979, ApJ, 187, 871

Kaufer, A., Stahl, O., Wolf, B., et al. 1997, A\& A, 320, 273

Kiriakidis, M., Fricke, K. J., \& Glatzel, W. 1993, MNRAS, 264, 50

Kiriakidis, M., Glatzel, W., \& Fricke, K. J. 1996, MNRAS, 281, 406

Lebreton, Y., Montalbán, J., Godart, M., Morel, P., Noels, A., \& Dupret, M. 2009, CoAst, 158, 277

Lefever, K., Puls, J., \& Aerts, C. 2007, A\&\&A, 463, 1093

Lefèvre, L., Marchenko, S. V., Moffat, A. F. J., et al. 2005, ApJ, 634, L109

Lovy, D., Maeder, A., Noels, A., \& Gabriel, M. 1984, A\&A, 133, 307

Lucy, L. B. 1976, ApJ, 206, 499

Mahy, L., Gosset, E., Baudin, F., et al. 2011, A\& 4 , 525, A101

Miglio, A. 2007, Ph.D. thesis, Université de Liège

Miglio, A., Montalbán, J., \& Dupret, M.-A. 2007, CoAst, 151, 48

Moravveji, E., Moya, A., \& Guinan, E. F. 2012b, ApJ, 749, 74

Moskalik, P. \& Dziembowski, W. A. 1992, A\&GA, 256, L5

Noels, A. \& Gabriel, M. 1981, A\& $A, 101,215$

Noels, A. \& Scuflaire, R. 1986, A\&A, 161, 125

Noels, A., Dupret, M.-A., \& Godart, M. 2008, Journal of Physics, Conf. Ser., 118, 012019

Ostrowski, J., Daszyńska-Daszkiewicz, J., \& Pamyatnykh, A. A. 2012, AN, 333, 946

Pamyatnykh, A. A. 1999. AcA, 49, 119

Pamyatnykh, A. A. 2007, CoAst, 150, 207

Puls, J., Urbaneja, M. A., Venero, R., Repolust, T., Springmann, U., Jokuthy, A., \& Mokiem, M. R. 2005, A\&A A, 435, 669

Rauw, G., Gosset, E., Manfroid, J., Vreux, J.-M., \& Claeskens, J.-F. 1996, A\&JA, 306, 783

Rogers, F. J. \& Iglesias, C. A. 1992, ApJS, 79, 507

Saio, H. 2009, CoAst, 158, 245

Saio, H. 2011, MNRAS, 412, 1814

Saio, H., Baker, N. H., \& Gautschy, A. 1998, MNRAS, 294, 622

Saio, H., Kuschnig, R., Gautschy, A., et al. 2006, ApJ, 650, 1111

Santolaya-Rey, A. E., Puls, J., \& Herrero, A. 1997, A\& A, 323, 488

Scuflaire, R. \& Noels, A. 1986, A\& A, 169, 185

Seaton, M. J., Yan, Y., Mihalas, D., \& Pradhan, A. K. 1994, MNRAS, 266, 805

Shibahashi, H. 1979, PASJ, 31, 87

Simon, N. R. \& Stothers, R. 1969, ApJ, 156, 377

Simón-Díaz, S. 2011, Bulletin de la Société Royale des Sciences de Liège, 80, 86

Simón-Díaz, S., Herrero, A., Uytterhoeven, K., Castro, N., Aerts, C., \& Puls, J. 2010, ApJ, 720, L174

Stothers, R. B. \& Chin, C.-W. 1993, ApJ, 408, L85

Townsend, R. H. D. \& MacDonald, J. 2006, MNRAS, 368, L57

Waelkens, C., Aerts, C., Kestens, E., Grenon, M., \& Eyer, L. 1998, A\&A, 330, 215

Ziebarth, K. 1970, ApJ, 162, 947 